\title{
Autosomal dominant vitreoretinochoroidopathy (ADVIRC)
}

\author{
NORMAN P. BLAIR, MORTON F. GOLDBERG, GERALD A. FISHMAN, AND \\ THOMAS SALZANO ${ }^{2}$
}

From the Department of Ophthalmology, College of Medicine, University of Illinois at Chicago, IL 60612, USA.

SUMMARY We report the second family recognised to have autosomal dominant vitreoretinochoroidopathy. The clinical features were (1) autosomal dominant inheritance; (2) peripheral, coarse pigmentary degeneration of the fundus for $360^{\circ}$, with a relatively discrete posterior border in the equatorial region (this finding may be pathognomonic); (3) superficial punctate yellowish-white opacities in the retina; (4) various vascular abnormalities; (5) breakdown of the blood-retinal barrier; (6) retinal neovascularisation; (7) vitreous abnormalities; and (8) choroidal atrophy. Visual reduction was mainly due to macular oedema or vitreous haemorrhage.

In 1982 Kaufman and associates reported for the first time a condition we termed autosomal dominant vitreoretinochoroidopathy. ${ }^{1}$ Its main features were: (1) an autosomal dominant hereditary pattern; (2) peripheral pigmentary retinopathy for $360^{\circ}$, with a discrete posterior boundary near the equator; (3) punctate whitish opacities in the retina; (4) vitreous cells and fibrillar condensation; (5) blood-retinal barrier breakdown; (6) retinal arteriolar narrowing and occlusion; (7) retinal neovascularisation; (8) choroidal atrophy; and (9) presenile cataracts. This unique combination of features indicated that this disorder was a distinct nosological entity. Although it is apparently rare, we have detected a second family with this disease in our clinic. This family is unrelated to the first and is reported here.

\section{Materials and methods}

Each available blood relative of the proband was examined with the following methods: (1) ocular and systemic history; (2) refraction; (3) external eye examination; (4) slit-lamp examination; (5) applanation tonometry; (6) dilated fundus examination with direct and indirect ophthalmoscopy; (7) screening for skeletal defects, including inspection and palpation of the palate, inspection of the spine, and examination for joint hyperextensibility and joint enlargement. In addition the following examinations were performed

Correspondence to Dr N. P. Blair, University of Illinois Eye and Ear Infirmary, 1855 West Taylor Street, Chicago, Il 60612, USA. on the proband, his parents, his siblings, and some of his other blood relatives: (1) Goldmann field examination; (2) fundus photography and fluorescein angiography; (3) vitreous fluorophotometry; (4) electroretinography; (5) Goldmann contact lens biomicroscopy and gonioscopy; (6) blood and urine testing for amino acids. Vitreous fluorophotometry was performed after an injection of $14 \mathrm{mg} / \mathrm{kg}$ of fluorescein sodium using a modified Haag-Streit $360^{\circ}$ slit-lamp or the Fluorotron Master (Coherent Radiation, Palo Alto) as previously described. ${ }^{23}$ The values $3 \mathrm{~mm}$ anterior to the chorioretinal peak were normalised to a blood fluorescein level of $10 \mu \mathrm{g} / \mathrm{ml}$.

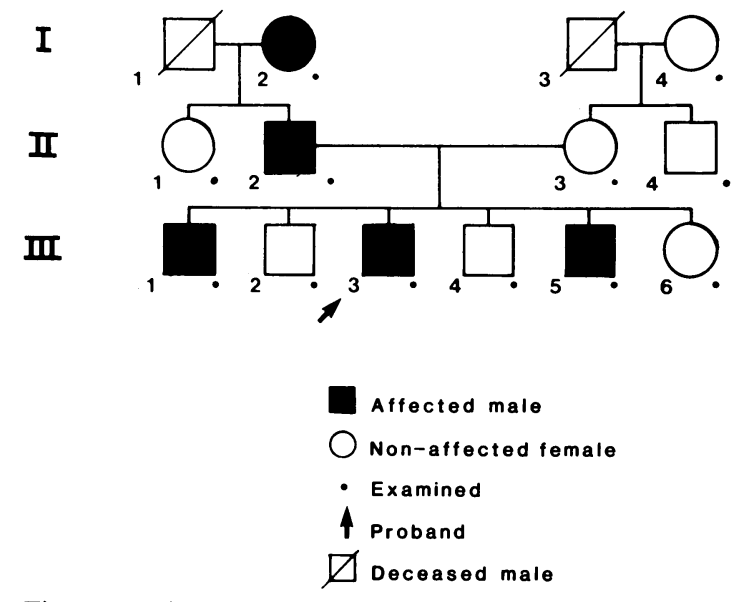

Fig. 1 Pedigree. 
Electroretinography was performed as previously described. ${ }^{4}$ Both white and chromatic stimuli were used to differentiate between rod and cone function. Both single and flicker stimuli were used.

\section{FAMILY STUDY}

See Fig. 1. No consanguinity was known. The paternal side of the family was of Italian origin, the maternal side of Polish. All family members had normal pregnancies and deliveries with the exception of the proband, who is described below. No systemic abnormalities were noted, including the results of careful clinical screening for skeletal abnormalities. The electroretinogram was normal in all family members. Urine and blood amino acid levels were normal, including those of ornithine.

\section{Case reports}

\section{Patient III-3}

The proband was first seen in 1976 as a 13-year-old white male because of recurrent vitreous haemorrhage in the right eye. Four months earlier he had received focal laser therapy for new vessels above the right optic nervehead by another ophthalmologist. He denied nyctalopia but did admit that his day vision was better than his night vision. He denied photophobia and colour vision problems. He had weighed $2100 \mathrm{~g}$ at birth and required supplemental oxygen for 3 days. The vision was $20 / 30$ in each eye without correction. The external examination and slit-lamp examination of the anterior segment were normal; both lenses were clear. The vitreous contained 3+ cells in each eye, but no optically empty space was observed. There appeared to be a posterior vitreous detachment in the left eye. The applanation pressures were 18 and $19 \mathrm{mmHg}$ in the right and left eyes respectively. Gonioscopy was unremarkable.

On funduscopic examination several abnormalities were seen bilaterally. There was marked, peripheral, coarse pigmentary mottling that extended for $360^{\circ}$ around the retinal periphery (Fig. 2). It had an abrupt border approximately at the equator, posterior to which the fundus looked relatively normal. Occasionally, the pigment was arranged in bone spicule configurations. Numerous yellowish-white dots were visible, which appeared to be in the region of the internal limiting membrane. These were most frequent in the area of the pigmentary changes. There were also many nodules on the internal limiting membrane in the area of the pigmentary disturbance. Occasional beading, sheathing, whitening, and occlusion of veins was seen, especially peripherally, where there was a generalised paucity of vessels. The retinal arteries appeared to be somewhat attenuated (Fig. 3A). There was greyish discoloration of the inner retina along the inferior temporal vascular arcades which was due to retinal oedema as determined by angiography. In the right eye there

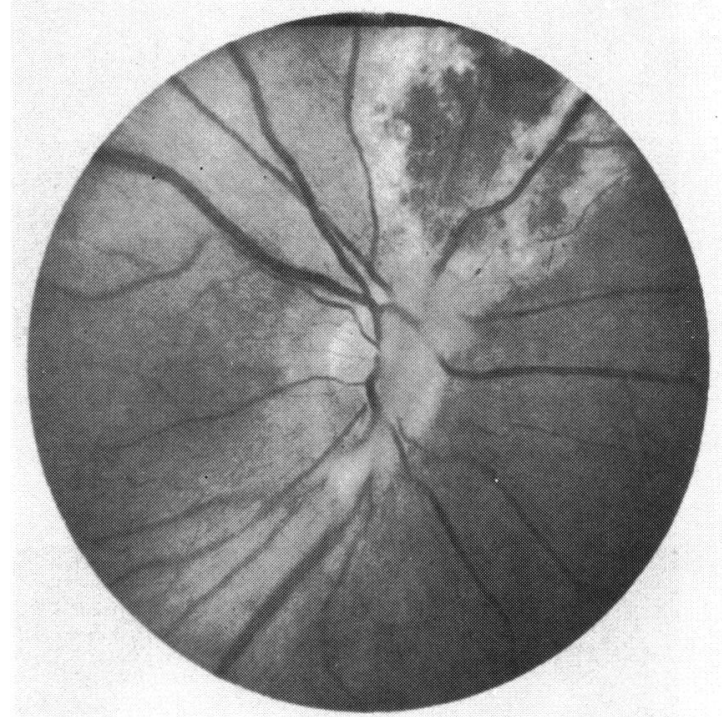

Fig. 3A

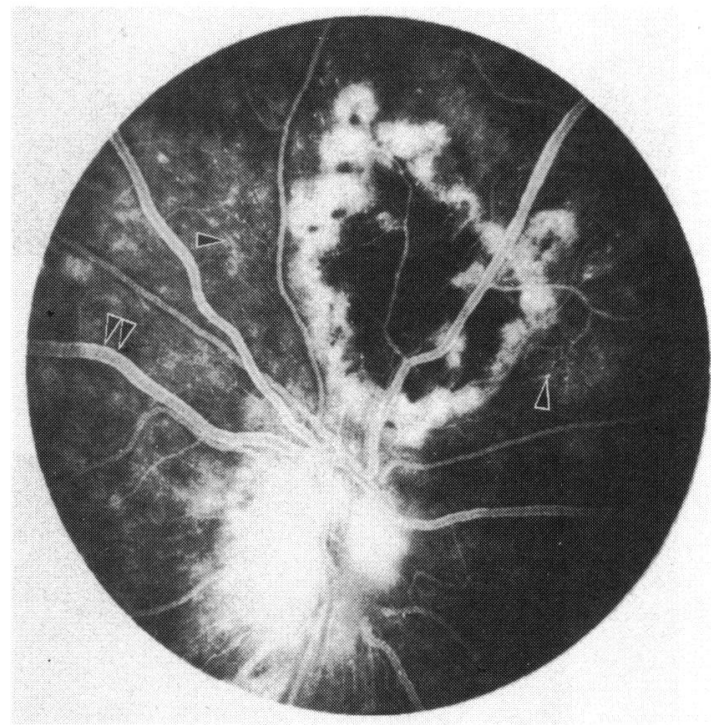

Fig. 3B

Fig. 3 Right optic disc region of proband, patient III-3. A. Prepapillary fibrous tissue and a scar from previous photocoagulation are seen. Mild arterial narrowing is present. Note the greyish discoloration of the retina along the inferotemporal arcade. B. The angiogram shows fine microvascular anomalies (arrowheads), particularly near the photocoagulation scar, and the disc shows late hyperfluorescence. Note staining of vein walls (double arrows). 


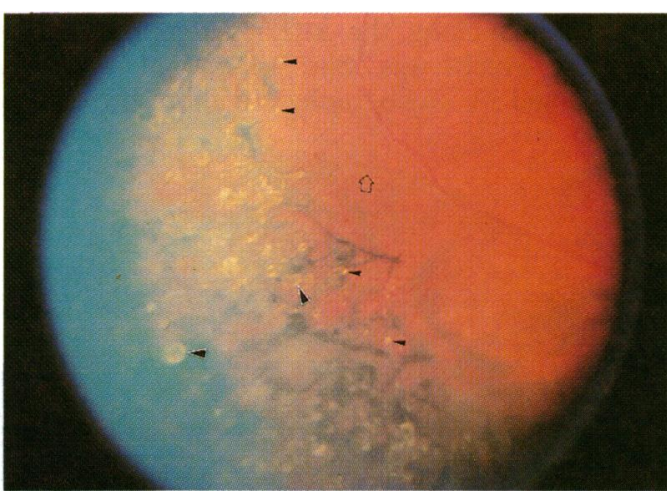

Fig. 2 Peripheral fundus of the proband, patient III-3. There is coarsely mottled retinal pigmentation that occasionally assumes a bone spicule pattern. The posterior boundary is relatively discrete. Multiple yellow dots (black arrowheads) and nodules (black and white arrowheads) are seen. Note the white vein (open arrow) which is occluded on the angiogram (see Fig. 5).

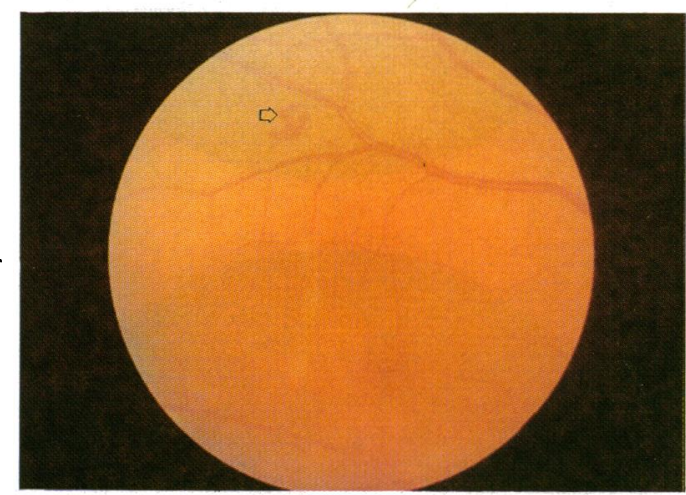

Fig. 4 Colour photograph of right macular area of proband, patient III-3. A focus of preretinal new vessels is present superotemporal to the macula near the arcade (arrow).

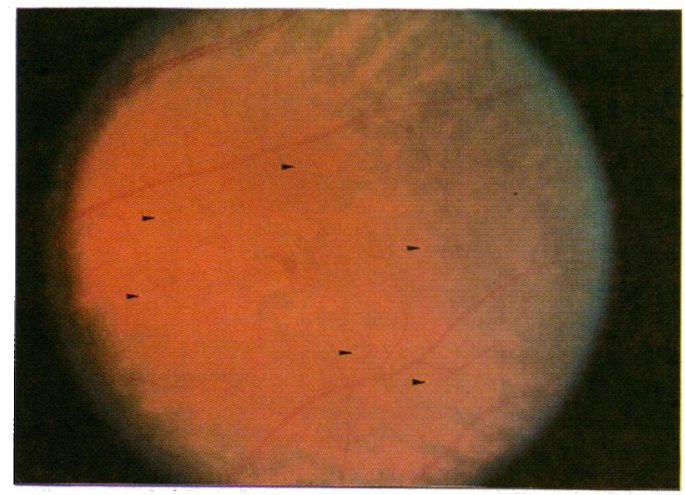

Fig. 7 Left macula of proband, patient III-3 in 1982. There is considerable pigmentary mottling in the foveal region. Note also the yellowish-white dots (arrowheads).

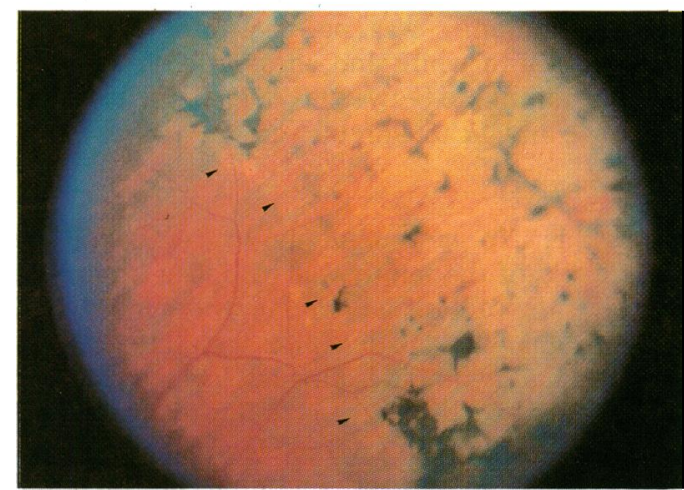

Fig. 11 Peripheral retina of patient III-5. Coarse pigmentary retinopathy with a relatively discrete posterior boundary (arrowheads) is seen. Some bone spicule pigmentary configurations are present.

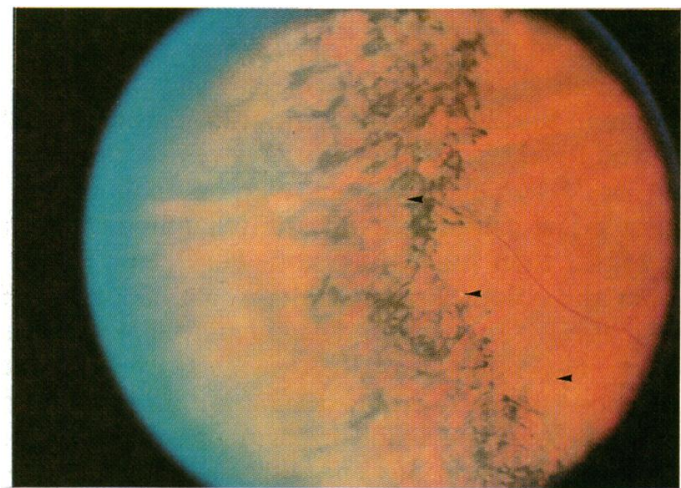

Fig. 12 Peripheral retina of patient III-1. Coarse pigmentary retinopathy with a discrete posterior boundary. Yellow dots are present (arrowheads). 


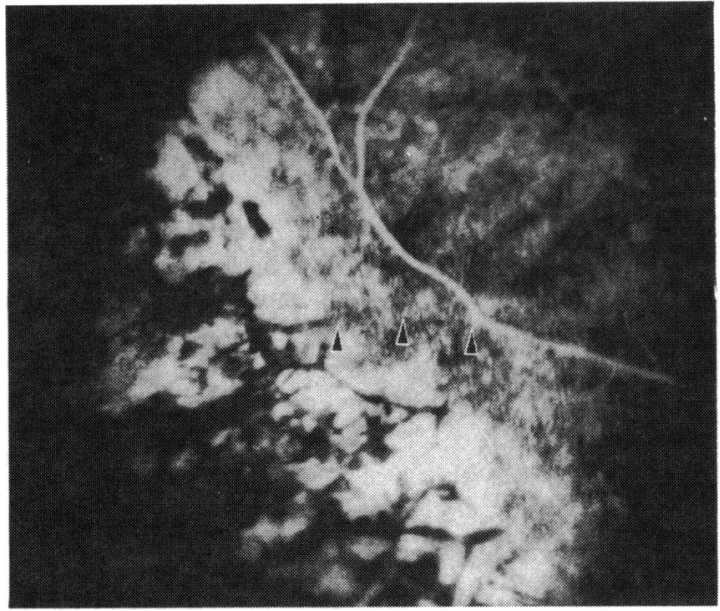

Fig. 5 Fluorescein angiogram of area seen in Fig. 2. There is a hyperfluorescent zone anterior to the equator due to window defects with superimposed blockage of fluorescence by pigment. More posteriorly, the choroidal fluorescence is finely irregular. A white retinal vein on the color photo (Fig. 2 ) is seen to be occluded on the angiogram (arrowheads).

was prepapillary fibrotic tissue, a scar superior to the disc due to previous photocoagulation, and fine microvascular abnormalities near the scar. Irregular staining of the vein walls was also seen (Fig. 3B). An area of retinal neovascularisation was seen superotemporal to the macula near the arcade (Fig. 4). Extensive vitreous haemorrhage was seen from the inferior arcade to the ora serrata extending from 5.00 to 8.00 o'clock.

Fluorescein angiography showed marked window defects peripherally with multiple foci of blocked fluorescence (Fig. 5). Posterior to the boundary of the marked pigmentary change there was fine pigmentary mottling. Subtle abnormalities of the calibre of the retinal capillaries were seen, including some at the perifoveal capillary net. There was late staining at the optic disc and in the region of the new vessels (Fig. 6). Mild depression of the visual fields was determined by Goldmann perimetry, and in addition there was a superior field defect in the right eye corresponding to the area of vitreous haemorrhage. The electroretinogram's photopic and scotopic responses were normal in amplitude and implicit times. It was initially thought that the patient might have some form of arrested retrolental fibroplasia, until examination of other family members revealed the hereditary nature of the disease.

The patient was next seen in June 1979. The vision at that time was $20 / 50 \mathrm{OU}$ with a correction of $-2 \cdot 25$ OD and -2.50 OS. The macula appeared to be displaced somewhat inferiorly and temporally in each eye. The vitreous haemorrhage in the right eye had

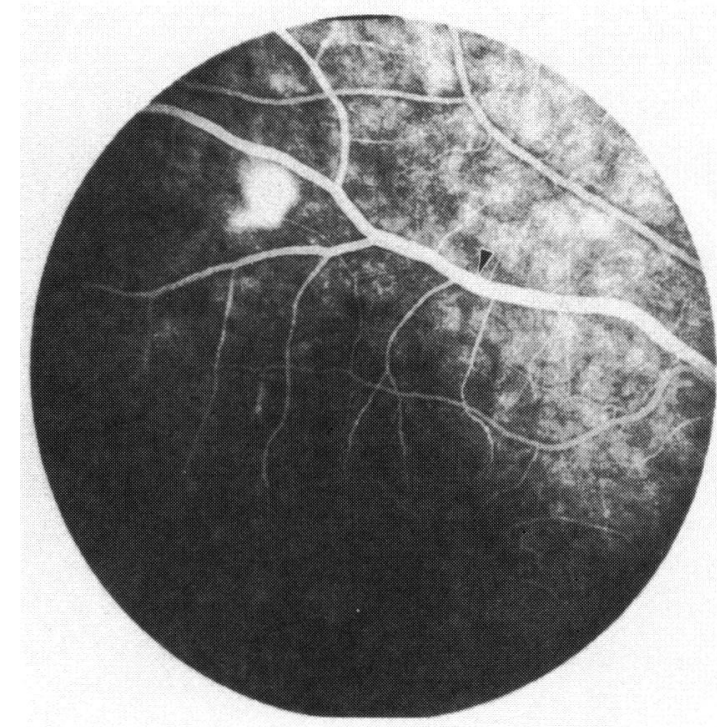

Fig. 6 Fluorescein angiogram of area shown in Fig. 4. There is leakage from the focus of retinal neovascularisation. Note also the slight venous beading (arrowhead). The macular pigmentation appears normal.

mostly resorbed, leaving a white intravitreal clot inferiorly. The focus of retinal neovascularisation along the superior temporal arcade was slightly enlarged as compared with the previous examination. A fluorescein angiogram showed that macular pigmentary changes had developed in the interim. There was moderate leakage along the arcades near the disc and some late disc staining.

In January 1982 the patient developed a black central spot in the central field of the left eye. The best corrected vision was $20 / 60(-4 \cdot 25+0 \cdot 50 \times 130)$ in the right eye and $20 / 300(-5 \cdot 00+0 \cdot 75 \times 75)$ in the left eye. There was a defect on Amsler grid testing OS. The anterior segment examination was changed only in that slight band keratopathy was noted at 3.00 o'clock near the limbus OS. On funduscopic examination there was increased pigmentary mottling at the maculas of both eyes (Fig. 7). Yellowish-white dots could be seen in the maculas, and considerable macular thickening, especially in the left eye, was noted. Fluorescein angiography showed marked leakage from the radial peripapillary capillaries and at the foveas (Fig. 8). On this examination some intravitreal bands were noted as well as vitreous condensation over the peripheral pigmented zone. A focus consistent with hypertrophy of the retinal pigment epithelium was observed at 4.00 o'clock in the left eye posterior to the boundary of the peripheral pigmented zone. As expected in a patient with marked leakage of fluorescein into the macula, 


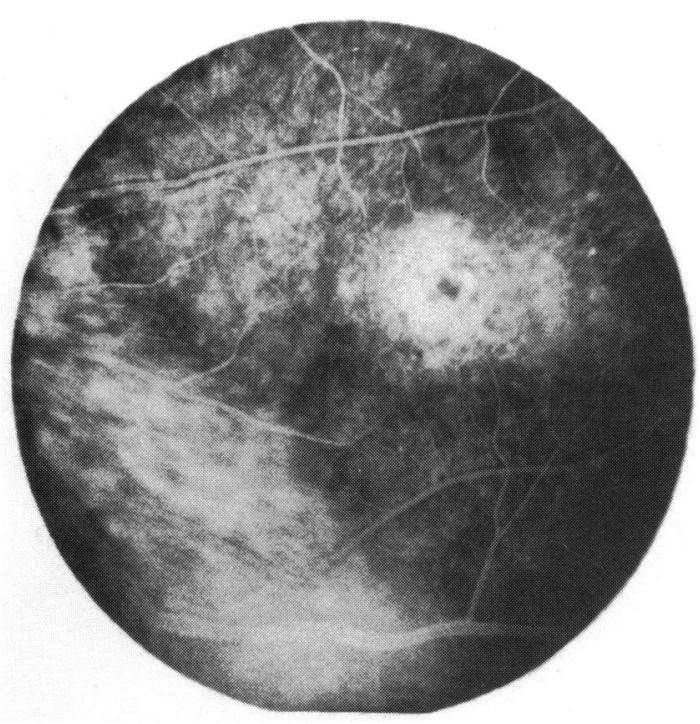

Fig. 8 Fluorescein angiogram of area near that seen in Fig. 7. Note marked leakage from the radial peripapillary capillaries and leakage into the macula.

the vitreous fluorophotometry readings were extremely elevated (Fig. 9). The electroretinogram was still normal (Fig. 10). A photic visual evoked response test was reduced in the left eye and also reduced, but within 2 standard deviations from normal, in the right eye. A pattern visual evoked response showed distinct and marked abnormalities in amplitude OU. The central scotoma spontaneously improved, but it has recurred twice. The vision was $20 / 200$ OS, with marked cystoid macular oedema and severe foveal pigmentary changes at the time of the last examination in December 1982.

\section{Patient III-5}

The 8-year-old brother of the proband was first examined in 1976. He had had an intermittent left exotropia since age 7 . He was otherwise asymptomatic; specifically, there was no history of any nyctalopia or colour vision problems. He was the product of a normal pregnancy and delivery and had no exposure to oxygen. The vision in the right eye was $20 / 40\left(-2 \cdot 50+0.50 \times 100^{\circ}\right)$ and in the left eye was $20 / 40\left(-3.25+0.75 \times 60^{\circ}\right)$. The extraocular motions were full, but there were 6 prism dioptres of exotropia at distance and 30 prism dioptres of exotropia at near. The external examination and slit-lamp examination of the anterior segment were normal. The applanation tensions were 13 and $14 \mathrm{mmHg}$ in the right and left eyes respectively. There were $4+$ pigmented cells in both vitreous bodies. Gonioscopy was normal. On funduscopic examination there was blotchy pigment mottling from the equator to the ora serrata with a relatively discrete posterior boundary (Fig. 11). There was perivascular pigmentation in some areas, and some of the pigmentation was arranged like bone spicules. Yellowish-white dots were seen in the region of the internal limiting membrane and were similar to those in his brother's eyes. The retinal arterioles were somewhat attenuated, and in some areas there were mild-calibre irregularities of the veins. There was prepapillary fibrous tissue without visible vessels in both eyes. Mild pigmentary changes at the macula were questionably present in each eye. The fluorescein angiogram showed a broad band of window defect with a fairly discrete posterior boundary near the equator. Coarse foci of blocked fluorescence were seen in this region and corresponded to the pigmented areas in the retina. Posteriorly there was fine mottling to the background choroidal fluorescence. The visual fields showed mild generalised depression. The electroretinogram was normal.

The patient was again examined in May 1982. At this time his vision was $20 / 40$ OD $(-7 \cdot 00+1 \cdot 50 \times 110)$ and $20 / 30$ OS $(-6 \cdot 50+0 \cdot 25 \times 120)$. Mild posterior embryotoxon was seen $\mathrm{OU}$. The fundus examination was similar to that at the previous examination except that occasional whitish nodules were seen on the internal limiting membrane, the large choroidal vessels could be seen even at the centre of the macula, and the retinal vessels over the peripheral pigmented zone were either not visible or white lines. Fluorescein angiography showed fine microaneurysmal changes at the perifoveal capillary ring, late leakage at the macula and optic nervehead, and the pigmentary changes seen previously. The vitreous fluorophotometry result was markedly elevated. The electroretinogram remained normal.

\section{Patient III-1}

The 22-year-old brother of the proband was first seen in 1982. He had no symptoms, no nyctalopia, no colour vision problems, and no history of prematurity. The vision was $20 / 30$ OD $(-9 \cdot 00+1.00 \times 70)$ and $20 / 25$ OS $(-9 \cdot 75+0 \cdot 75 \times 120)$. The external examination, applanation tonometry, and slit-lamp examinations were normal except for slight peripheral posterior cortical opacities in each lens. With slitlamp biomicroscopy $1+$ vitreous dots, translucent lines, and posterior vitreous detachments were seen. On indirect ophthalmoscopy the posterior segment examination showed a band of pigmentary degeneration extending for $360^{\circ}$ in each eye, as in his brothers (Fig. 12). Yellow-white dots near the internal limiting membrane were observed, but no nodules were seen. There was increased visibility of the choroidal vasculature even at the macula of each eye. A fluorescein angiogram revealed late leakage from the discs, 


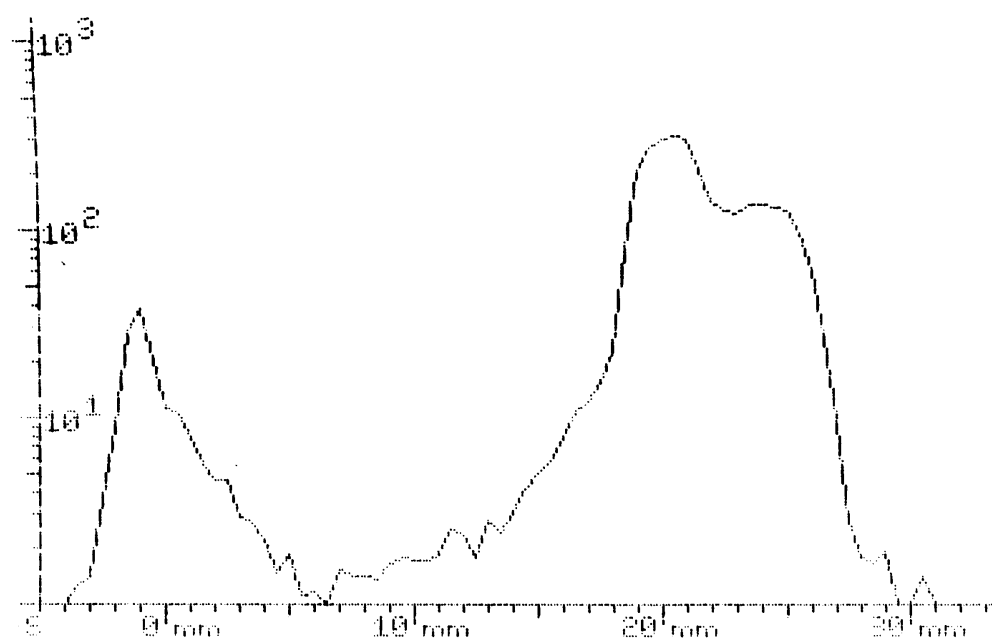

Fig. 9A

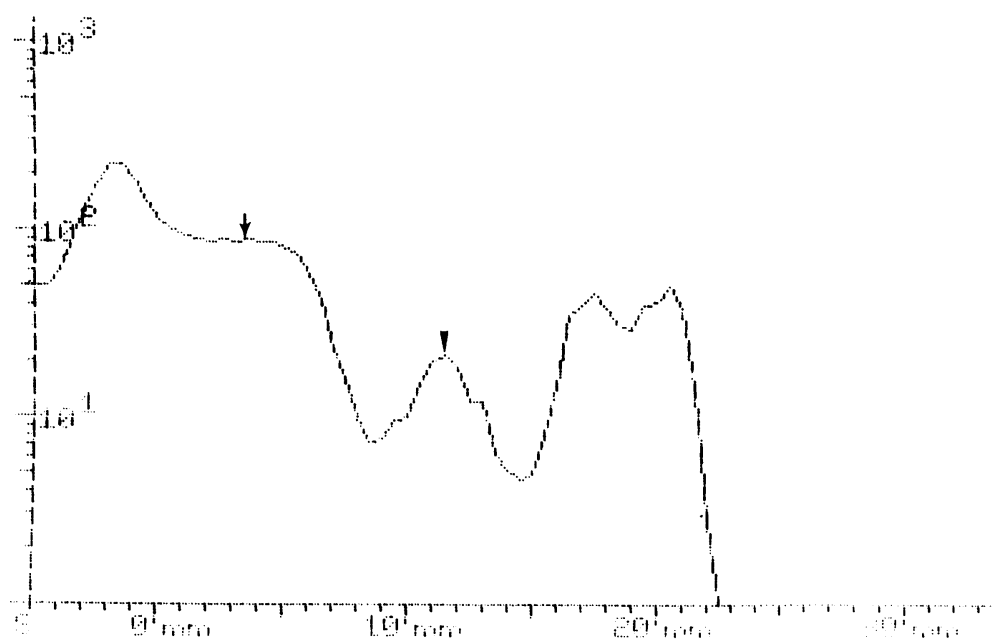

Fig. 9 Vitreous fluorophotometry recordings. A. Plot of vitreous fluorescein concentration versus distance from a normal subject recorded 62 minutes after injection of $14 \mathrm{mg} / \mathrm{kg}$ of sodium fluorescein. Note. from left to right: chorioretinal peak, vitreous, lens peak, anterior chamber. The value $3 \mathrm{~mm}$ anterior to the chorioretinal peak is $4 \cdot 7$ $\mathrm{ng} / \mathrm{ml}$, which is normal. B. A similar scan from the proband, patient III-3 in 1982 taken 13 minutes after sodium fluorescein. Note the abnormal contour of the vitreous fluorescein concentration with high levels (arrow) near the chorioretinal peak and another peak in the anterior vitreous (arrowhead). The value $3 \mathrm{~mm}$ anterior to the chorioretinal peak is $92 \mathrm{ng} / \mathrm{ml}$, over 9 times the concentration normally found 1 hour after injection.

Fig. 9B

peripheral window defects, and areas of blocked fluorescence. The retinal arterioles appeared to be slightly attenuated. There was slight depression on visual field testing in both eyes. The vitreous fluorophotometry results were markedly elevated in both eyes. The electroretinogram was normal.

\section{Patient II-2}

The 45-year-old father of the proband denied any ocular symptoms. His vision was $20 / 20$ OU $(-3.50$ $\mathrm{OU})$. The external examination, applanation tonometry, and slit-lamp examination were unremarkable except for mild punctate cortical flecks in the lenses. The gonioscopic examination was normal. Biomicroscopy of the vitreous using a Goldmann lens showed slight vitreous degeneration and syneresis, no vitreous cells, and posterior vitreous detachment $O U$. Peripherally, fine yellow dots were seen in the region of the internal limiting membrane in some areas. The fundus examination revealed several peripheral foci of pigmented blotches $\mathrm{OU}$. The fluorescein angiogram, visual fields, vitreous fluorophotometry, and electroretinogram were normal.

\section{Patient I-2}

The 76-year-old paternal grandmother of the proband was asymptomatic. She refused to come to the hospital, so she was examined at home. An external examination by flashlight was unremarkable except for a few cortical spokes and mild nuclear sclerosis of both lenses. The dilated fundus examination with direct and indirect ophthalmoscopy showed several 


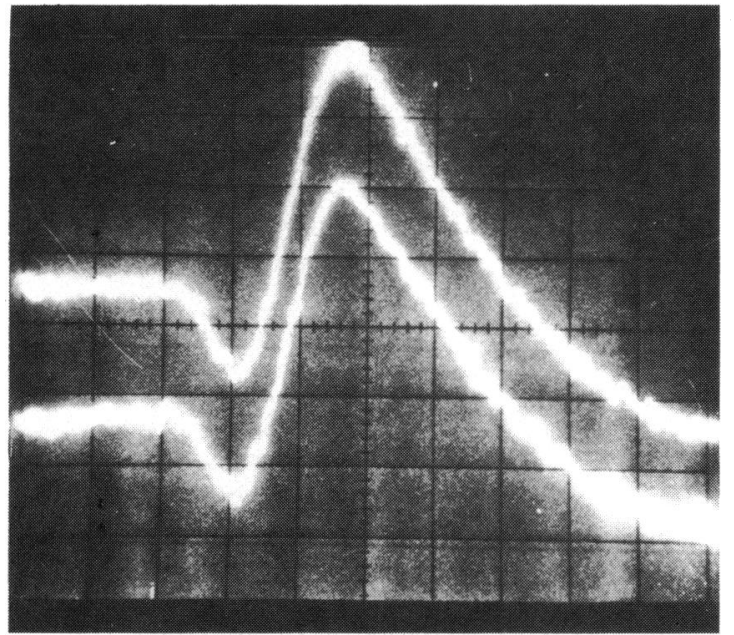

Fig. 10 Electroretinogram of proband, patient III-3 in 1982. The recording was taken under scotopic conditions with a white light. It is normal in amplitude and timing.

peripheral pigmented blotches and a few yellow dots which seemed more discrete, more yellow, smaller, and rounder than drusen. There was mild choroidal atrophy as evidenced by increased visibility of the large choroidal vessels even in the macula. No macular drusen were seen. There was a prominent $360^{\circ}$ peripapillary conus in each eye with a tilted disc in the left eye.

\section{Discussion}

We have described the second family with autosomal dominant vitreoretinochoroidopathy. Our recognition of a second affected family so shortly after seeing the original family suggests that the condition may be more common than we had assumed. Although peripheral pigmentary retinopathy was a prominent feature of this disease, visual loss appears to be related mainly to macular oedema due to breakdown of the blood-retinal barrier or vitreous haemorrhage due to retinal neovascularisation. Cystoid macular oedema has been severe and recurrent in the probands of both this pedigree and of the one we initially reported. ${ }^{1}$

When all of the features of this ocular disease are present, diagnosis should be straightforward because it appears that this combination of findings is unique. The major characteristics which are useful in differentiating this condition from others are as follows: (1) Familial pattern. This feature, for the most part, excludes a wide variety of retinal vascular diseases that could produce breakdown of the blood-retinal barrier and retinal neovascularisation. (2) Peripheral pigmentary disturbance. We know of no other con- dition where there is a broad band of marked pigmentary degeneration, which extends from a discrete posterior border in the equatorial region to the ora serrata for $360^{\circ}$. It may be a pathognomonic sign of this disease. (3) Yellow-white dots. These dots appeared to be in the retina or on the internal limiting membrane and not at the level of the pigment epithelium. They sometimes appeared to glisten, and diameter was approximately $30 \mu \mathrm{m}$. They were usually seen peripherally in the region of the coarse pigmentary disturbance, but also could be seen in the posterior pole. Similar foci are known to occur in a variety of retinal degenerative conditions. ${ }^{5}$ (4) Prominent vascular abnormalities. These included vascular leakage, arteriolar attenuation, venous beading, venous sheathing, venous occlusion, focal venous staining, microaneurysm formation, and retinal neovascularisation. (5) Absence of significant nyctalopia. This effectively rules out various forms of congenital stationary night blindness, such as fundus albipunctatus, and progressive rod-cone dystrophies, such as choroideremia and retinitis pigmentosa. (6) Normal electroretinogram. This eliminates most forms of retinitis pigmentosa, Goldmann-Favre disease, and congenital retinoschisis. (7) Absence of prominent systemic features. This excludes a variety of syndromes which potentially could be confused with this one, such as Stickler syndrome ${ }^{6}$ and the metabolic tapetoretinal degenerations. ${ }^{7}$

The conditions which we considered most seriously in the differential diagnosis were autosomal dominant cystoid macular oedema and snowflake deposition in hereditary vitreoretinal degeneration. Autosomal dominant cystoid macular oedema is a recently described condition characterised, as its name suggests, by autosomal dominant inheritance and cystoid macular oedema. ${ }^{89}$ Additional abnormalities include leakage from the optic disc capillaries, mild peripheral pigmentary retinopathy, ${ }^{10}$ vitreous opacities, subnormal electrooculography values, and normal electroretinograms at least during the initial stages. In our family the peripheral pigmentary abnormalities were severe and were more frequent than was frank macular oedema. In autosomal dominant cystoid macular oedema the peripheral pigmentary changes are not very prominent, and are much less frequent than are the macular changes. Furthermore the reported pigmentary changes did not have the characteristics seen in our cases, where there was extensive peripheral pigmentary mottling for $360^{\circ}$ with a relatively discrete posterior boundary.

Hereditary vitreoretinal degeneration with snowflake degeneration was described by Hirose et al. ${ }^{5}$ It is characterised by autosomal dominant inheritance and a sequential evolution of changes consisting of (1) extensive white-with-pressure, (2) snowflake 
degeneration, (3) sheathing of retinal vessels and pigmentation, (4) increased pigmentation and disappearance of vessels. Fibrillar vitreous degeneration progressed along with the fundus changes. Five of their 15 cases developed rhegmatogenous retinal detachment. The peripheral pigmentation, yellowwhite dots (snowflakes), and vascular changes are reminiscent of the condition we have seen. However, snowflake degeneration is not characterised by intense pigmentation having a discrete posterior boundary. Hirose et al. also did not describe significant macular oedema, microvascular changes, or retinal neovascularisation. Prominent white-withpressure, the evolution of changes that they described, and rhegmatogenous retinal detachment were not seen in our patients. We also excluded Bietti's tapetoretinal degeneration with marginal corneal dystrophy, ${ }^{11}$ the disease described by Wagner, ${ }^{12}$ and familial exudative vitreoretinopathy ${ }^{13}$ because of the marked vascular decompensation and characteristic pigment distribution seen in our patients. However, marked vascular leakage occasionally occurs in the latter condition. ${ }^{14}$

We have included the father and paternal grandmother of the proband as being affected by the condition, even though they did not have the extensive changes seen in the proband and his 2 brothers. We consider these 2 to have minimally expressed disease. However, their mild changes possibly could be nonspecific. If so, it would be impossible to determine conclusively the hereditary pattern of the disease in this family.

It is difficult to determine the primary site of the underlying disease process in this condition. The vitreous is involved with mild degenerative changes, vitreous cells, vitreous strands, and early posterior vitreous detachment. The retina is involved with vascular changes and pigmentary abnormalities. The choroid is abnormal because some choriocapillaris atrophy must be present for the large choroidal vasculature to be visible in the foveal region. It may be that the primary sites of involvement are the retinal pigment epithelium and the retinal vessels, and that the other changes are secondary, including presenile cataract. ${ }^{1}$ This would explain the abnormal vitreous fluorophotometry and normal electroretinogram results in our patients.

In summary, we present the second family with autosomal dominant vitreoretinochoroidopathy, a disease with protean manifestations in the ocular posterior segment. So far this disease has been recognised only in Chicago, Illinois, although the 2 kindreds were not related. Visual loss appears to be related to vitreous haemorrhage or macular oedema and may be severe. Variable expressivity may be present, as in most dominant traits, and suggests that examination of family members may aid in establishing the diagnosis in questionable or minimally affected cases. Because the term autosomal dominant vitreoretinochoroidopathy is cumbersome, we suggest the acronym ADVIRC as a convenient name until the precise pathogenesis and primary anatomical locus of involvement are established.

This study was supported in part by Public Health Service Research Grant EY 03106 (Dr Blair), Ophthalmic Research Center Core Grant EY 1792 and Training Grant EY 7038 from the National Eye Institute, Bethesda, Maryland, and by an unrestricted grant from Research to Prevent Blindness, Inc, NY, NY.

Thanks are expressed to Reuben Matalon, MD, for performing the amino acid analyses, to Marlene Heneghan for secretarial assistance, and to Maxine Gere for editorial services.

Presented in part at the International Symposium on Retinal Diseases, San Diego, California, October 1982.

\section{References}

1 Kaufman SJ, Goldberg MF, Orth DH, Fishman GA, Tessler H, Mizuno K. Autosomal dominant vitreoretinochoriodopathy. Arch Ophthalmol 1982; 100: 272-8.

2 Cunha-Vaz JG. Vitreous fluorophotometry. In: Cunha-Vaz JG, ed. The blood-retinal barriers. New York: Plenum, 1980: 195-210.

3 Zeimer RC, Blair NP, Cunha-Vaz JG. Vitreous fluorophotometry for clinical research. I. Description and evaluation of a new fluorophotometer. Arch Ophthalmol in press.

4 Fishman GA, Fishman M, Maggiano J. Macular lesions associated with retinitis pigmentosa. Arch Ophthalmol 1977; 95: 798-803.

5 Hirose T, Lee KY, Schepens CL. Snowflake degeneration in hereditary vitreoretinal degeneration. Am J Ophthalmol 1974; 77: $143-53$

6 Blair NP, Albert DM, Liberfarb RM, Hirose T. Hereditary progressive arthroophthalmopathy of Stickler. Am J Ophthalmol 1979; 88: 876-88.

7 François J. Metabolic tapetoretinal degenerations. Surv Ophthalmol 1982; 26: 293-333.

8 Deutman AF, Pinckers AJLG, Aan de Kerk AL. Dominantly inherited cystoid macular edema. Am J Ophthalmol 1976; 82: $540-8$.

9 Fishman GA, Goldberg MF, Trautmann JC. Dominantly inherited cystoid macular edema. Ann Ophthalmol 1979; 11: 21-7.

10 Notting JGA, Pinckers AJLG. Dominant cystoid macular dystrophy. Am J Ophthalmol 1977; 83: 234-241.

11 Bagolini B, Ioli-Spada G. Bietti's tapetoretinal degeneration with marginal corneal dystrophy. Am J Ophthalmol 1968; 65: 53-60.

12 Wagner H. Ein bisher unbekanntes Erbleiden des Auges (Degeneratio hyaloideo-retinalis hereditaria), beobachtet im Kanton Zurich. Klin Monatsbl Augenheilkd 1938; 100: 840-57.

13 Criswick VG, Schepens CL. Familial exudative vitreoretinopathy. Am J Ophthalmol 1969; 68: 578-94.

14 Swanson D, Rush P, Bird AC. Visual loss from retinal oedema in autosomal dominant exudative vitreoretinopathy. $\mathrm{Br} J$ Ophthalmol 1982; 66: 627-9. 